\title{
Hadron Mass Effects in Power Corrections to Event Shapes*
}

\author{
Vicent Mateu ${ }^{\dagger}$ \\ Center for Theoretical Physics, Massachusetts Institute of Technology, Cambridge, MA 02139, \\ USA \\ IFIC, UVEG - CSIC, Apartado de Correos 22085, E-46071, Valencia, Spain \\ E-mail: mateu@mit.edu

\section{lain W. Stewart} \\ Center for Theoretical Physics, Massachusetts Institute of Technology, Cambridge, MA 02139, \\ USA \\ E-mail: iainsemit.edu \\ Jesse Thaler \\ Center for Theoretical Physics, Massachusetts Institute of Technology, Cambridge, MA 02139, \\ USA \\ E-mail: jthaleremit.edu
}

\begin{abstract}
We study the effect of hadron masses on the leading power correction of dijet event-shape distributions. We define the transverse velocity operator, that describes the effects of hadron masses. It depends on the "transverse velocity" $r$, which is different from one only for non-vanishing hadron masses. We find that hadron-mass effects in general break universality. However we provide a simple method to identify universality classes of event shapes with a common power correction. We also compute the anomalous dimension of the power correction and the structure of the corresponding Wilson coefficient, finding a nontrivial result.
\end{abstract}

Xth Quark Confinement and the Hadron Spectrum,

October 8-12, 2012

TUM Campus Garching, Munich, Germany

\footnotetext{
*IFIC/13-01, LPN13-009, MIT-CTP 4432

† Speaker.
} 


\section{Introduction}

Event shapes have been crucial to pin down the structure of QCD. In the recent years the subject has attracted a lot of attention due to the very precise extraction of the strong coupling constant $\alpha_{s}$ from fits to the tail of the thrust distribution [1,2,3] and moments of the thrust distribution $[4,5]^{1}$. Excellent reviews on event shapes are $[7,8]$, where the definition of the most commonly used can be found.

Even though event shapes are infrared safe observables, they receive sizable corrections from hadronization effects. In the tail of the distribution these effects are known as power corrections and are suppressed by inverse powers of the center-of-mass energy $Q$. The first studies of power corrections were inspired on renormalon techniques. The dispersive approach of Dokshitzer and Webber $[9,10,11]$ replaces $\alpha_{s}$ by an effective coupling below some cutoff scale. Within this approach it was found that the leading power correction for different event shapes were proportional to one another, with a calculable coefficient [9, 12]. Later, Salam and Wicke [13] pointed out using the flux-tube model that hadron mass effects break that universality.

A different approach to power corrections is based on the factorization properties of QCD at very high energies. The shape function introduced in $[14,15]$ parametrizes nonperturbative corrections and describes the tail power corrections to any order. Moreover, within this approach nonperturbative parameters are expressed as matrix elements of QCD operators. Lee and Sterman [16] showed that the factorization approach predicts the same universality relations as the dispersive model. Recently it has been shown in the Soft-Collinear Effective Theory framework (SCET for short) $[17,18,19,20,21]$ framework that hadron masses break universality [22].

\section{Event shapes in the dijet limit}

An event shape $e$ is an observable defined on the kinematical properties of the final-state hadrons (energy and three-momentum). For our purposes we will use the dimensionful quantities $p_{\perp}=\left|\vec{p}_{\perp}\right|$ (transverse momentum) and $m^{\perp}=\sqrt{p_{\perp}^{2}+m^{2}}$ (transverse mass), and the dimensionless variables $\eta=-\ln \tan (\theta / 2)$ (pseudorapidity) and $y=1 / 2 \ln \left[\left(E+p_{z}\right) /\left(E-p_{z}\right)\right]$ (rapidity). Here $\theta$ refers to the polar angle from the $\hat{z}$ axis, which we take to be aligned with the thrust axis. The velocity of a particle is $v=|\vec{p}| / E$, and we define the transverse velocity as $r=p_{\perp} / m^{\perp}$. For massless particles $p_{\perp}=m^{\perp}, y=\eta$ and $r=v=1$, but the equalities no longer hold for non-zero masses.

Dijet event shapes tend to zero for a configuration of two narrow back-to-back jets plus soft radiation (that is, for a dijet configuration) and vice versa. In the dijet limit $e \ll 1$ one can expand $e=\bar{e}+\mathscr{O}\left(e^{2}\right)$, where $\bar{e}$ is in general simpler than $e$. In particular one can always write

$$
\bar{e}=\frac{1}{Q} \sum_{i \in X} m_{i}^{\perp} f_{e}\left(r_{i}, y_{i}\right)
$$

where the function $f_{e}$ is specific for a given event shape. In this limit one can derive factorization theorems for the differential cross section, which are very convenient to perform resummation of singular logarithms to all orders in perturbation theory, and to identify power corrections [23].

\footnotetext{
${ }^{1}$ See also [6] for a determination using the Heavy Jet Mass distribution.
} 


\section{Power Corrections}

Using SCET one can derive a factorization formula for the singular cross section:

$$
\frac{\mathrm{d} \sigma_{s}}{\mathrm{~d} e}=\int \mathrm{d} \ell \frac{\mathrm{d} \hat{\sigma}_{s}}{\mathrm{~d} e}\left(e-\frac{\ell}{Q}\right) F_{e}(\ell)[1+\mathscr{O}(e)]
$$

Here $\mathrm{d} \hat{\sigma}_{s} / \mathrm{d} e$ refers to the partonic singular distribution whereas $\mathrm{d} \sigma_{s} / \mathrm{d} e$ is the nonperturbative singular distribution. $\mathrm{d} \hat{\sigma}_{s} / \mathrm{d} e$ diverges as $\log ^{i}(e) / e$ for $e \rightarrow 0$ and hence dominates in the dijet limit. $F_{e}$ is the shape function, which contains nonperturbative corrections (plus some perturbative terms [22]).

In the tail of the distribution, defined by the condition $Q e \gg \Lambda_{\mathrm{QCD}}$ the shape function can be expanded in inverse powers of $\ell \gg \Lambda_{\mathrm{QCD}}$ :

$$
F_{e}(\ell)=\delta(\ell)-\delta^{\prime}(\ell) \Omega_{1}^{e}(\mu)+\mathscr{O}\left(\frac{\alpha_{s} \Lambda_{\mathrm{QCD}}}{\ell^{2}}\right)+\mathscr{O}\left(\frac{\Lambda_{\mathrm{QCD}}^{2}}{\ell^{3}}\right),
$$

Here $\Omega_{1}^{e}(\mu)$ is a nonperturbative matrix element defined by

$$
\Omega_{1}^{e}=\left\langle 0\left|\bar{Y}_{\bar{n}}^{\dagger} Y_{n}^{\dagger}(Q \hat{e}) Y_{n} \bar{Y}_{\bar{n}}\right| 0\right\rangle .
$$

and $\hat{e}$ is the event-shape operator defined as

$$
\hat{e}|X\rangle=e(X)|X\rangle,
$$

with $|X\rangle$ the state of a configuration of particles in the final state of a given event, and $e(X)$ the value of the event shape for that configuration. $Y$ and $\bar{Y}$ are Wilson lines of soft gluon fields in the light-like directions $n$ and $\bar{n}$.

Using Eq. (3.2) in (3.1) one finds at leading order that the effect of the power corrections is to shift the distribution:

$$
\frac{\mathrm{d} \sigma}{\mathrm{d} e}=\frac{\mathrm{d} \hat{\sigma}}{\mathrm{d} e}-\frac{\Omega_{1}^{e}}{Q} \frac{\mathrm{d}}{\mathrm{d} e} \frac{\mathrm{d} \hat{\sigma}}{\mathrm{d} e}+\ldots=\frac{\mathrm{d} \hat{\sigma}}{\mathrm{d} e}\left(e-\frac{\Omega_{1}^{e}}{Q}\right)+\ldots
$$

A similar result is found in the dispersive model $[9,10,11]$.

\section{Universality}

In order to study the effects of hadron masses on power corrections we need to express the event-shape operator $\hat{e}$ in terms of quantum fields. Following the approach of Refs. [16, 23] we find that it can be written in terms of the energy-momentum tensor. Let us start by introducing the "transverse velocity operator", defined by its action on a state $|X\rangle$ :

$$
\hat{\mathscr{E}}_{T}(r, y)|X\rangle=\sum_{i \in X} m_{i}^{\perp} \delta\left(r-r_{i}\right) \delta\left(y-y_{i}\right)|X\rangle .
$$

It is important to use rapidity $y$ and not pseudorapidity $\eta$ as only the former transforms in an additive way under a longitudinal boost. In Ref. [22] it was shown that $\hat{\mathscr{E}}_{T}$ can be expressed solely in terms of the energy-momentum tensor. Now the event-shape operator can be written as

$$
\hat{e} \equiv \frac{1}{Q} \int_{-\infty}^{+\infty} \mathrm{d} y \int_{0}^{1} \mathrm{~d} r f_{e}(r, y) \hat{\mathscr{E}}_{T}(r, y) .
$$




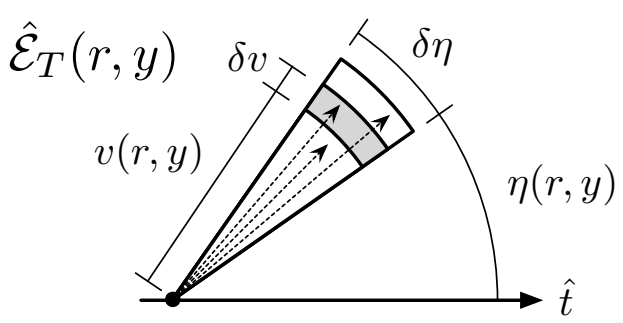

Figure 1: Graphical representation of the transverse velocity operator. Measurements are made with respect to the thrust axis $\hat{t}$. The arrows correspond to particles with lengths given by the particle velocities. Shading indicates which particles are measured by the operator. Note that the velocity $v(r, y)$ and pseudo-rapidity $\eta(r, y)$ are functions of the transverse velocity $r$ and rapidity $y$.

According the leading power correction is written in terms of a double integral:

$$
\Omega_{1}^{e}=\int_{-\infty}^{+\infty} \mathrm{d} y \int_{0}^{1} \mathrm{~d} r f_{e}(r, y)\left\langle 0\left|\bar{Y}_{\bar{n}}^{\dagger} Y_{n}^{\dagger} \mathscr{E}_{T}(r, y) Y_{n} \bar{Y}_{\bar{n}}\right| 0\right\rangle
$$

As depicted in Fig. 1, the transverse velocity operator $\hat{\mathscr{E}}_{T}(r, y)$ involves a spheroid that expands in both space and time with a finite velocity $v$, and it measures the total transverse mass for particles in an infinitesimal interval in both $\eta$ and the velocity $v$ (or equivalently an infinitesimal interval in $y$ and $r$ ). Following Ref. [16] one can apply boost transformations along the thrust axis to figure out in which cases universality is preserved. Both the vacuum $|0\rangle$ and the Wilson lines are boost invariant, however under a boost of rapidity $y^{\prime}$ the transverse velocity operator transforms as follows:

$$
U\left(y^{\prime}\right) \hat{\mathscr{E}}_{T}(r, y) U\left(y^{\prime}\right)^{\dagger}=\hat{\mathscr{E}}_{T}\left(r, y+y^{\prime}\right) .
$$

Therefore, choosing $y^{\prime}=-y$ in Eq. (4.3) we can write the leading power correction as

$$
\Omega_{1}^{e}=c_{e} \int_{0}^{1} \mathrm{~d} r g_{e}(r) \Omega_{1}(r),
$$

with

$$
\Omega_{1}(r) \equiv\left\langle 0\left|\bar{Y}_{\bar{n}}^{\dagger} Y_{n}^{\dagger} \hat{\mathscr{E}}_{T}(r, 0) Y_{n} \bar{Y}_{\bar{n}}\right| 0\right\rangle
$$

a universal nonperturbative function, and

$$
c_{e}=\int_{-\infty}^{+\infty} \mathrm{d} y f_{e}(1, y), \quad g_{e}(r)=\frac{1}{c_{e}} \int_{-\infty}^{+\infty} \mathrm{d} y f_{e}(r, y) .
$$

The function $g_{e}(r)$ encodes all hadron mass effects. Defining

$$
\Omega_{1}^{g_{e}} \equiv \int_{0}^{1} \mathrm{~d} r g_{e}(r) \Omega_{1}(r),
$$

one can write $\Omega_{1}^{e}=c_{e} \Omega_{1}^{g e}$, which implies that the leading power correction for two event shapes $e_{1}$ and $e_{2}$ are proportional to each other if $g_{e_{1}}(r)=g_{e_{2}}(r)$. We will denote the set of all event shapes 


\begin{tabular}{l|llccc}
$c_{e}$ & $\tau$ & $\tau_{2}$ & $\tau_{(a)}$ & $C$ & $\rho_{ \pm}$ \\
\hline Common & 2 & 2 & $\frac{2}{1-a}$ & $3 \pi$ & 1
\end{tabular}

Table 1: Expression for the $c_{e}$ coefficients for various dijet event shapes. Since $c_{e}$ are defined using $f_{e}(1, y)$, they have the same value in each universality class. Here $\tau$ refers to thrust, $\tau_{2}$ to 2 -Jettiness, $\tau_{a}$ to angularities, $C$ to the C-parameter and $\rho_{ \pm}$to the hemisphere masses.

with the same $g_{e}(r)$ function as a universality class. All event shapes with the same universality class have the same power correction up to a calculable factor.

The coefficients $c_{e}$ match exactly the classic universality prefactors obtained when hadron masses are neglected $[24,9,10,25,14,26,27,28,16]$. Table 1 summarizes the values of the $c_{e}$ coefficients for the most common event shapes.

\section{Mass schemes and universality classes}

The standard definition of an event shape involves in general both the energy and the magnitude of the momentum of the final state hadrons (on top of the directions). In an experimental environment one has access to a limited amount of information. Although directions are easily measured, in general one has information on the energy deposited by the particle in the detector, but not on its momentum. If the particle is identified the momentum can be of course reconstructed, but that is not always possible.

The E-scheme is an alternative definition for any event shape in a way that only the experimentally accessible information is used. Specifically one makes the following replacement in the event-shape definition:

$$
\vec{p}_{i} \rightarrow \frac{E_{i}}{\left|\vec{p}_{i}\right|} \vec{p}_{i}
$$

It is easy to show $[13,22]$ that all event shapes defined in the E-scheme belong to the same universality class: the E-scheme class.

Analogously one can define the P-scheme class by the replacement $E_{i} \rightarrow\left|\vec{p}_{i}\right|$. Although event shapes defined in that way do not belong to the same class, they have nevertheless similar power corrections [13,22].

We define two additional schemes: the R-scheme, in which one replaces $\eta$ by y in the P-scheme expression of $\bar{e}$ and then uses $e^{R}=\bar{e}^{R}$; and the J-scheme, in which one sets $r=1$ in the R-scheme expression.

Table 2 summarizes which universality class event shapes (in various schemes) belong to.

\section{Anomalous Dimension and Matching Coefficient}

The expression of $\Omega_{1}(r)$ in Eq. (4.6) is only a formal definition. In general, matrix elements in a quantum field theory which do not directly correspond to an observable have to be defined within a scheme. In Ref. [22] the anomalous dimension of $\Omega_{1}(r)$ in the $\overline{\mathrm{MS}}$ scheme was computed at one loop. The diagrams giving a non-vanishing contribution are shown in Figs. 2 and 3. It turns out 


\begin{tabular}{l|c|l} 
Class & $g(r)$ & Event shape \\
\hline Jet Mass class $\left(\Omega_{1}^{0}\right.$ or $\left.\Omega_{1}^{\rho}\right)$ & 1 & $\rho_{ \pm}, \tau_{2}, \tau^{J}, \tau_{(a)}^{J}, C^{J}$ \\
E-scheme class $\left(\Omega_{1}^{1}\right.$ or $\left.\Omega_{1}^{E}\right)$ & $r$ & $\tau_{(a)}, \tau^{E}=\tau_{2}^{E}, C^{E}, \rho_{ \pm}^{E}, \tau^{R}, \tau_{(a)}^{R}, C^{R}, \rho_{ \pm}^{R}$ \\
$r^{n}$ class $\left(\Omega_{1}^{n}\right)$ & $r^{n}$ & generalized angularities $\tau_{(n, a)}[22]$ \\
\hline Thrust class $\left(\Omega_{1}^{g_{\tau}}\right)$ & $g_{\tau}(r)$ & $\tau, \rho_{ \pm}^{P}, \tau_{2}^{P}$ \\
$C$-parameter class $\left(\Omega_{1}^{g C}\right)$ & $g_{C}(r)$ & $C$ \\
$r^{2}$ class $\left(\Omega_{1}^{2}\right)$ & $r^{2}$ & $\tau_{(2, a)}, \tau_{(a \rightarrow-\infty)}^{P}$
\end{tabular}

Table 2: Event shape classes with a universal first power correction parameter $\Omega_{1}^{g_{e}}$. For a given event shape, the full power correction is $\Omega_{1}^{e}=c_{e} \Omega_{1}^{g_{e}}$. Superscripts $E, P, J$, and $R$ correspond to event shapes measured in the E-, P-, J-, and R-schemes, respectively.

that only non-abelian terms contribute, and one finds

$$
\mu \frac{\mathrm{d}}{\mathrm{d} \mu} \Omega_{1}(r, \mu)=\left[-\frac{\alpha_{s} C_{A}}{\pi} \ln \left(1-r^{2}\right)\right] \Omega_{1}(r, \mu) .
$$

Interestingly, the anomalous dimension is $r$-dependent, although there is no mixing for different values of $r$. This implies that hadron masses play an essential role.

From Eq. (6.1), for two renormalization scales $\mu$ and $\mu_{0}$ of comparable size one has

$$
\Omega_{1}^{e}(\mu)=\Omega_{1}^{e}\left(\mu_{0}\right)+\frac{\alpha_{s}\left(\mu_{0}\right) C_{A}}{\pi} \ln \left(\frac{\mu}{\mu_{0}}\right) \Omega_{1}^{e, \ln }\left(\mu_{0}\right),
$$

with

$$
\Omega_{1}^{e, \ln }\left(\mu_{0}\right) \equiv-\int \mathrm{d} r \ln \left(1-r^{2}\right) c_{e} g_{e}(r) \Omega_{1}\left(r, \mu_{0}\right) .
$$

Given that $\Omega_{1}(r, \mu)$ runs, one expects that the expansion of the shape function in Eq. (3.2) should involve a non-trivial matching coefficient. Hence we write

$$
F_{e}(\ell)=\delta(\ell)+\int \mathrm{d} r C_{1}^{e}(\ell, r, \mu) c_{e} g_{e}(r) \Omega_{1}(r, \mu)+\mathscr{O}\left(\frac{\Lambda_{\mathrm{QCD}}^{2}}{\ell^{3}}\right) .
$$

Consistency with Eq. (6.1) requires that the matching coefficient at one loop has the following form

$$
\begin{aligned}
C_{1}^{e}(\ell, r, \mu)= & -\delta^{\prime}(\ell)+\frac{C_{A} \alpha_{s}(\mu)}{\pi} \ln \left(1-r^{2}\right) \frac{\mathrm{d}}{\mathrm{d} \ell}\left(\frac{1}{\mu}\left[\frac{\mu}{\ell}\right]_{+}\right) \\
& +\frac{\alpha_{s}(\mu)}{\pi} \delta^{\prime}(\ell) d_{1}^{e}(r)+\mathscr{O}\left(\alpha_{s}^{2}\right) .
\end{aligned}
$$

The structure of Eq. (6.5) was checked by an explicit calculation in Ref. [22].

\section{Conclusions}

We have studied hadron-mass effects for event shapes in the SCET formalism. These effects have been expressed in terms of QCD matrix elements. Our results show that hadron masses break universality although within certain classes it is still preserved. We have computed the oneloop running of the power correction, finding a nontrivial anomalous dimension and matching coefficient. We largely confirm the results of Ref. [13]. 

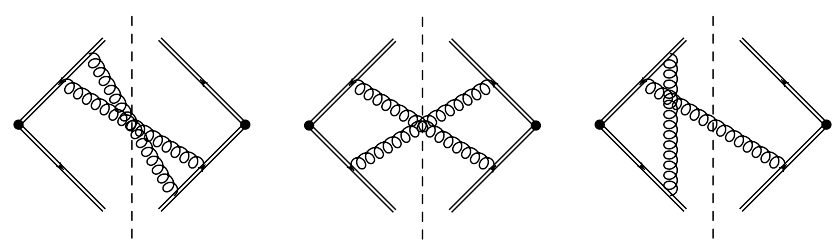

Figure 2: Independent emission diagrams with Abelian and non-Abelian contributions. The four additional diagrams obtained by a horizontal flip or complex conjugation are not shown.
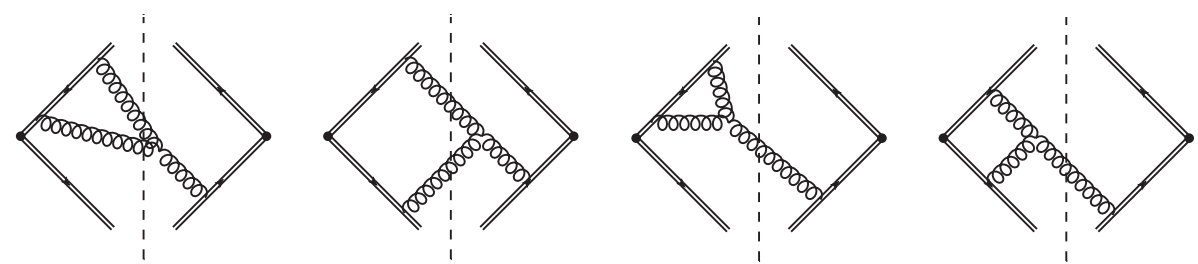

Figure 3: Triple gluon Y-diagrams for the $\mathscr{O}\left(\alpha_{s}^{2}\right)$ correction to $\Omega_{1}(r)$. The twelve additional diagrams obtained by a horizontal flip or complex conjugation are not shown. Diagrams with all three gluons coupled to Wilson lines of the same direction vanish.

\section{Acknowledgments}

This work was supported by the offices of Nuclear and Particle Physics of the U.S. Department of Energy (DOE) under grant numbers DE-FG02-94ER-40818 and DE-FG02-05ER-41360, the European Community's Marie-Curie Research Networks under contract PITN-GA-2010-264564 (LHCphenOnet) and by MISTI global seed funds. VM is supported by a Marie Curie Fellowship under contract PIOF-GA-2009-251174.

\section{References}

[1] T. Becher and M. D. Schwartz, A Precise determination of $\alpha_{s}$ from LEP thrust data using effective field theory, JHEP 07 (2008) 034, [0 803 . 0342].

[2] R. Abbate, M. Fickinger, A. H. Hoang, V. Mateu, and I. W. Stewart, Thrust at $N^{3} L L$ with Power Corrections and a Precision Global Fit for $\alpha_{s}\left(m_{Z}\right)$, Phys. Rev. D83 (2011) 074021, [1006.3080].

[3] T. Gehrmann, G. Luisoni, and P. F. Monni, Power corrections in the dispersive model for a determination of the strong coupling constant from the thrust distribution, 1210.6945.

[4] T. Gehrmann, M. Jaquier, and G. Luisoni, Hadronization effects in event shape moments, Eur. Phys. J. C67 (2010) 57-72, [0911.2422].

[5] R. Abbate, M. Fickinger, A. H. Hoang, V. Mateu, and I. W. Stewart, Precision Thrust Cumulant Moments at $N^{3} L L, 1204.5746$.

[6] Y.-T. Chien and M. D. Schwartz, Resummation of heavy jet mass and comparison to LEP data, JHEP 08 (2010) 058, [1 005.1644 ].

[7] M. Dasgupta and G. P. Salam, Event shapes in e+e-annihilation and deep inelastic scattering, J.Phys. G30 (2004) R143, [hep-ph/0312283].

[8] S. Kluth, Tests of quantum chromo dynamics at e+e-colliders, Rept. Prog. Phys. 69 (2006) 1771-1846, [hep-ex/0603011]. 
[9] Y. L. Dokshitzer and B. R. Webber, Calculation of power corrections to hadronic event shapes, Phys. Lett. B352 (1995) 451-455, [hep-ph/9504219].

[10] Y. L. Dokshitzer, G. Marchesini, and B. R. Webber, Dispersive Approach to Power-Behaved Contributions in QCD Hard Processes, Nucl. Phys. B469 (1996) 93-142, [hep-ph/9512336].

[11] Y. L. Dokshitzer, A. Lucenti, G. Marchesini, and G. Salam, On the universality of the Milan factor for 1 / Q power corrections to jet shapes, JHEP 9805 (1998) 003, [hep-ph/9802381].

[12] R. Akhoury and V. I. Zakharov, On the universality of the leading, $1 / Q$ power corrections in QCD, Phys. Lett. $B 357$ (1995) 646-652, [hep-ph/9504248].

[13] G. P. Salam and D. Wicke, Hadron masses and power corrections to event shapes, JHEP 05 (2001) 061, [hep-ph/0102343].

[14] G. P. Korchemsky and G. Sterman, Power corrections to event shapes and factorization, Nucl. Phys. B555 (1999) 335-351, [hep-ph/9902341].

[15] G. P. Korchemsky and S. Tafat, On power corrections to the event shape distributions in QCD, JHEP 10 (2000) 010, [hep-ph/ 0007005$].$

[16] C. Lee and G. Sterman, Momentum flow correlations from event shapes: Factorized soft gluons and soft-collinear effective theory, Phys. Rev. D75 (2007) 014022, [hep-ph/ 0611061 ].

[17] C. W. Bauer, S. Fleming, and M. E. Luke, Summing Sudakov logarithms in B $\rightarrow$ X/s gamma in effective field theory, Phys. Rev. D 63 (2001) 014006, [hep-ph/ 0005275 ].

[18] C. W. Bauer, S. Fleming, D. Pirjol, and I. W. Stewart, An effective field theory for collinear and soft gluons: Heavy to light decays, Phys. Rev. D 63 (2001) 114020, [hep-ph / 0011336$].$

[19] C. W. Bauer and I. W. Stewart, Invariant operators in collinear effective theory, Phys. Lett. B 516 (2001) 134-142, [hep-ph/0107001].

[20] C. W. Bauer, D. Pirjol, and I. W. Stewart, Soft-Collinear Factorization in Effective Field Theory, Phys. Rev. D65 (2002) 054022, [hep-ph/ 010904 5].

[21] C. W. Bauer, S. Fleming, D. Pirjol, I. Z. Rothstein, and I. W. Stewart, Hard scattering factorization from effective field theory, Phys. Rev. D 66 (2002) 014017, [hep-ph/ 0202088 ].

[22] V. Mateu, I. W. Stewart, and J. Thaler, Power Corrections to Event Shapes with Mass-Dependent Operators, Phys.Rev. D87 (2013) 014025, [1209.3781].

[23] C. W. Bauer, S. P. Fleming, C. Lee, and G. F. Sterman, Factorization of e+e- Event Shape Distributions with Hadronic Final States in Soft Collinear Effective Theory, Phys.Rev. D78 (2008) 034027, [0801.4569].

[24] G. P. Korchemsky and G. Sterman, Nonperturbative corrections in resummed cross-sections, Nucl. Phys. B437 (1995) 415-432, [hep-ph/9411211].

[25] Y. L. Dokshitzer and B. Webber, Power corrections to event shape distributions, Phys.Lett. B404 (1997) 321-327, [hep-ph/9704298].

[26] A. V. Belitsky, G. P. Korchemsky, and G. Sterman, Energy flow in QCD and event shape functions, Phys. Lett. $B 515$ (2001) 297-307, [hep-ph/ 0106308 ].

[27] C. F. Berger and G. Sterman, Scaling rule for nonperturbative radiation in a class of event shapes, JHEP 09 (2003) 058, [hep-ph/ 0307394 ].

[28] C. F. Berger and L. Magnea, Scaling of power corrections for angularities from dressed gluon exponentiation, Phys.Rev. D70 (2004) 094010, [hep-ph/ 0407024 ]. 